\title{
Temporal evolution of the magnetically active eclipsing binary DV Psc
}

\author{
S. Palafouta and K. Gazeas \\ National and Kapodistrian University of Athens, Department of Physics, \\ Section of Astrophysics, Astronomy and Mechanics, GR 15784 Zografos, \\ Athens, Greece, (E-mail: spalafouta@gmail.com)
}

Received: October 31, 2019; Accepted: January 24, 2020

\begin{abstract}
The existence of magnetic activity on the eclipsing binary DV Psc has been known for almost two decades. However, until recently, no evidence of periodic behaviour relevant to this activity had been found. In this study, long-term photometric observations of DV Psc are used to analyze the system's magnetic activity, seek a possible magnetic cycle and determine orbital and physical parameters. The combination of photometric and spectroscopic observations results in a unified model that describes the system over time in terms of variable spot activity. New times of minimum light are determined and an accurate astronomical ephemeris and updated O-C diagram are constructed for a total span of 19 years (1997-2017). The intense magnetic activity, as indicated by strong asymmetries in the light curves (O' Connell effect), and the periodic variation of the $\mathrm{O}-\mathrm{C}$ diagram are combined to explain the system's behaviour. The existence of a third body, orbiting the eclipsing binary in an eccentric orbit, as well as a magnetic cycle are the most likely scenario.
\end{abstract}

Key words: stars: activity - binaries: close - binaries: eclipsing

\section{Introduction}

The eclipsing binary DV Psc was discovered to be a variable by Robb et al. (1999). It belongs to the class of RS CVn systems, exhibiting strong magnetic activity. Several studies of this target exist in the literature (Lu et al., 2001; Parimucha et al., 2010), some of which resulting in contradicting conclusions (Zhang \& Zhang, 2007; Pi et al., 2012, 2014).

\section{The current study}

Our observations confirm the highly variable magnetic activity, which changes the light curve dramatically on a small time scale. The observed light curve asymmetries affect the calculation of times of minima, resulting in a variable $\mathrm{O}$ $\mathrm{C}$ diagram (Fig.1). Times of minimum light determined from our observations, as well as those available in the literature, are used for the O-C analysis in the current study. We found both components to be slightly evolved dwarfs, located 
well inside the Main Sequence. The cyclic behavior of the O-C diagram suggests a possible third body, orbiting the binary, as well as the presence of prominent magnetic activity, both of which affecting the times of minima. The orbit of the third body is estimated to have a period of $9.79 \pm 0.60 \mathrm{yr}$, while the magnetic cycle of the system is estimated to last $14.74 \pm 0.84 \mathrm{yr}$.
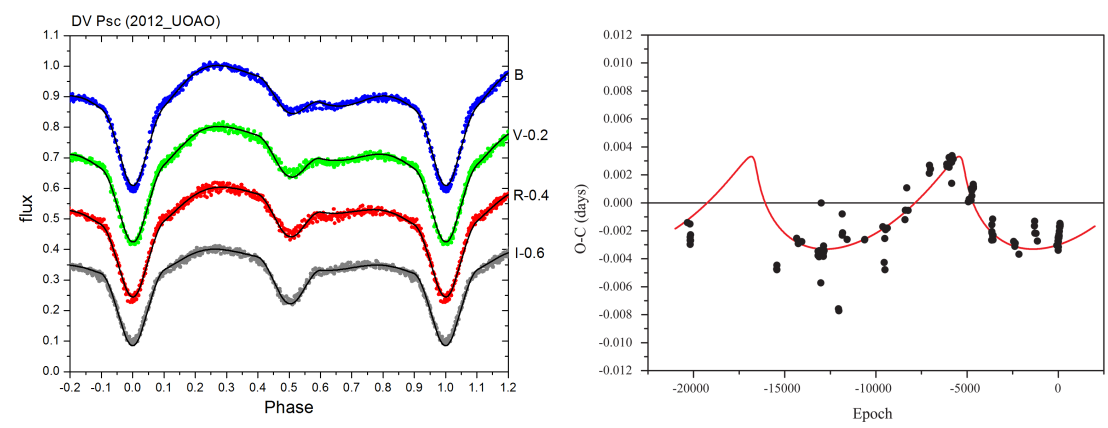

Figure 1. An example of multi-band photometric variability of DV Psc for data obtained in 2012. Solid curves represent theoretical models. The right panel shows the cyclic behavior of the $\mathrm{O}-\mathrm{C}$ diagram extracted from the times of minimum light.

\section{References}

Lu, W., Rucinski, S. M., \& Ogłoza, W., Radial Velocity Studies of Close Binary Stars. IV. 2001, Astron. J., 122, 402, DOI: 10.1086/321131

Parimucha, Š., Pribulla, T., Rucinski, S., et al., Preliminary Analysis of DV Psc: A Spotted, Short-Period Eclipsing Binary. 2010, in Astronomical Society of the Pacific Conference Series, Vol. 435, Binaries - Key to Comprehension of the Universe, ed. A. Prša \& M. Zejda, 99

Pi, Q., Zhang, L., Li, Z., Sang, H., \& Zhu, Z., Photometric study of the short-period RS CVn eclipsing binary DV Psc. 2012, arXiv e-prints, arXiv:1209.1862

Pi, Q.-f., Zhang, L.-Y., Li, Z.-m., \& Zhang, X.-l., Magnetic Activity and Orbital Period Variation of the Short-period Eclipsing Binary DV Psc. 2014, Astron. $J .$, 147, 50, DOI: 10.1088/0004-6256/147/3/50

Robb, R. M., Wagg, J., Berndsen, A., \& Desroches, L., Photometry of the Eclipsing Binary Star GSC $0008324=1$ RXS J001309+053550. 1999, Information Bulletin on Variable Stars, 4800

Zhang, X. B. \& Zhang, R. X., The physical nature of the short-period RS CVn system DV Psc. 2007, Mon. Not. R. Astron. Soc., 382, 1133, DOI: 10.1111/j.1365-2966.2007.12293.x 